\title{
The ZeRos OF THE THIRD DERIVATIVE OF BesSEL FUnCTIONS OF ORDER LESS THAN ONE
}

\author{
Lee Lorch
}

\begin{abstract}
Each positive zero of $J_{\nu}^{\prime \prime \prime}(x), 0<\nu<1,0<x<j_{\nu 1}$, is shown to be monotonic in $\nu$. The first such decreases to 0 , the second increases to $\sqrt{3}$, as $\nu \uparrow 1$. They occur when and only when $\nu_{0}<\nu<1, \nu_{0}=0.755578 \ldots$, and are precisely two in number. When $\nu=\nu_{0}, J_{\nu}^{\prime \prime \prime}(x)$ has a double zero. The double zeros of $J_{\nu}^{\prime \prime \prime}(x)$, for all real $\nu$, have been studied by M. K. Kerimov and S. L. Skorokhodov [USSR Comput. Maths. Phys., v.25, 6 (1985), pp. 101-107; Soviet Math. Dokl. v.33 (1986), pp. 650-652]. For $\nu=\nu_{0}$, the results coincide. The remaining (simple) positive zeros of $J_{\nu}^{\prime \prime \prime}(x), \nu>0$, without the restriction $0<x<j_{\nu 1}$, have been shown to be increasing functions of $\nu$ [L. Lorch and P. Szego, Methods Appl. Anal. 2 (1995), pp. 103-111]. Some inequalities for the zeros studied here are established.
\end{abstract}

\section{Introduction and some formulae}

We shall discuss here the positive zeros, $j_{\nu k}^{\prime \prime \prime}$, when they exist, of the t'lird derivative of the Bessel function $J_{\nu}(x), 0<\nu<1$, which precede the first positive zero $j_{\nu 1}$ of $J_{\nu}(x)$. The main purpose is to show that there are two such when $\nu_{0}<\nu<1$, $\nu_{0}=0.755578 \ldots$, none when $0<\nu<\nu_{0}$, and $(\S 4)$ as $\nu$ increases to 1 , that the first such decreases to 0 while the second increases to $\sqrt{3}=j_{11}^{\prime \prime \prime}$ (cf. (1.3)).

This is by way of completing results found in [7], albeit by an entirely different method. There it was shown that all other positive zeros of $J_{\nu}^{\prime \prime \prime}(x), \nu>0$, are increasing functions of $\nu$.

In addition, as in [7], some inequalities for these zeros are established, here for $\nu_{0}<\nu<1$ (cf. $\S 5$ ).

M. K. Kerimov and S. L. Skorokhodov [3,4] have studied double zeros of various Bessel functions and their derivatives, emphasizing computational aspects. For $\nu=\nu_{0}$, the result here coincides with their corresponding result which was obtained from different software.

The function $J_{\nu}(x)$ is defined by the alternating series

$$
J_{\nu}(x):=\frac{1}{2^{\nu}} \sum_{k=0}^{\infty} \frac{(-1)^{k} x^{2 k+\nu}}{4^{k} k ! \Gamma(\nu+k+1)} .
$$

It satisfies the Bessel differential equation $[8, \S 3.1(8)$, p. 40]

$$
x^{2} y^{\prime \prime}+x y^{\prime}+\left(x^{2}-\nu^{2}\right) y=0
$$

Received May 11, 1994, revised September 29, 1994.

1991 Mathematics Subject Classification. 33A40.

Key words and phrases: Bessel functions, zeros, monotonicity, inequalities.

This work received partial support from the Natural Sciences and Engineering Research Council of Canada. 
and consequently also

$$
x^{3} y^{\prime \prime \prime}+x\left(x^{2}-\nu^{2}-2\right) y^{\prime}+\left(3 \nu^{2}-x^{2}\right) y=0 .
$$

It satisfies also the recursion formula $[8, \S 3.2(4), \mathrm{p} .45]$

$$
x J_{\nu}^{\prime}(x)=\nu J_{\nu}(x)-x J_{\nu+1}(x) .
$$

The principal results are formulated in Theorem 4.1, supplemented by Theorem 4.2. Leading up to them are various technical preliminaries gathered in $\S 2$. Also required are convexity and similar properties of $J_{\nu}^{\prime \prime \prime}(x)$ in $0<x<j_{\nu 1}$, when $0<\nu<1$. These provide the content of $\S 3$ and establish that $J_{\nu}^{\prime \prime \prime}(x)$ cannot vanish more than twice in $0<x<j_{\nu 1}$ when $0<\nu<1$.

This done, the main results $(\S 4)$ follow on showing that any such crossing of the $x$-axis by the graph of $J_{\nu}^{\prime \prime \prime}(x)$ is lowered when $\nu$ is increased.

Finally, $\S 5$ furnishes various inequalities for these zeros. For ease of notation, the zeros $j_{\nu k}^{\prime \prime \prime}$ of $J_{\nu}^{\prime \prime \prime}(x)$ which occur in $0<x<j_{\nu 1}$ will be denoted by $\lambda$, a function of $\nu$, with $k$ fixed. On occasion, the pair of zeros which are found in $0<x<j_{\nu 1}$ for appropriate $\nu$ will be denoted by $\lambda_{1}, \lambda_{2}$, with $\lambda_{1}<\lambda_{2}$.

\section{Auxiliary results}

Gathered here are a number of miscellaneous results, principally inequalities, which are required for the monotonicity proofs offered in $\S 4$.

Theorem 2.1. If $0<\nu<1$, then

$$
J_{\nu}^{\prime \prime \prime}(0+)=+\infty \quad \text { and } \quad J_{\nu}^{\prime \prime \prime}(\nu \sqrt{3})>0
$$

Proof. The first assertion is evident from the alternating series obtained by differentiating (1.1) three times. For the second, put $x^{2}=3 \nu^{2}$ in the differential equation (1.3). Then

$$
\left(3 \nu^{2}\right)^{3 / 2} J_{\nu}^{\prime \prime \prime}(\nu \sqrt{3})+\nu \sqrt{3}\left(\nu^{2}-1\right) J_{\nu}^{\prime}(\nu \sqrt{3})=0 .
$$

Now $[8, \S 15.3(3), \mathrm{p} .486],{j^{\prime}}_{\nu 1}^{2}>\nu^{2}+2 \nu>3 \nu^{2}$, so that $J_{\nu}^{\prime}(\nu \sqrt{3})>0$ where $j_{\nu 1}^{\prime}$, is the first positive zero of $J_{\nu}^{\prime}(x)$. The second assertion is now evident.

We shall need also $[7,(4.2)]$

$$
\lambda>j_{\nu 1}>2.4 \ldots, \quad 0<\nu<0.7552 .
$$

Theorem 2.2. If $0<\nu<1$ and $\lambda<j_{\nu 1}$, then

$$
1-\nu<\lambda^{2}<3 \nu^{2}<\nu^{2}+2 \nu<j_{\nu 1}^{2} \text {. }
$$

Proof. The first inequality has been established in [7, (4.3)]. The last is cited in the proof of (2.1), leaving $\lambda^{2}<3 \nu^{2}$ to be proved.

It is already known [7, (2.6)] that $\lambda^{2}<\nu^{2}+2,0<\nu<1$. Thus, the remaining inequality would be a consequence of the fact that $J_{\nu}^{\prime \prime \prime}(x)$ is an increasing function of $x$ in $3 \nu^{2} \leq x^{2} \leq \nu^{2}+2$, in view of (2.1). 
Now, from (1.1), differentiated four times,

$$
\begin{aligned}
& 384 \Gamma(\nu+3) 2^{\nu} x^{4-\nu} J_{\nu}^{(4)}(x) \\
& >384 \nu\left(\nu^{2}-1\right)\left(\nu^{2}-4\right)(\nu-3)-96 \nu\left(\nu^{2}-1\right)(\nu+2)^{2} x^{2} \\
& \quad+12(\nu+1)(\nu+2)(\nu+3)(\nu+4) x^{4}-(\nu+4)(\nu+5)(\nu+6) x^{6} \\
& \quad:=f_{1}\left(x^{2}\right) .
\end{aligned}
$$

For $0<\nu<1$, the cubic $f_{1}(x)$ has at most two positive roots according to Descartes' Rule of Signs. It has either two such or none. Hence, if $f_{1}\left(3 \nu^{2}\right)>0$ and $f_{1}\left(\nu^{2}+2\right)>0$, then $f_{1}(x)>0,3 \nu^{2} \leq x \leq \nu^{2}+2$, and it would follow that $J_{\nu}^{(4)}(x)>0,3 \nu^{2} \leq x^{2} \leq \nu^{2}+2$, thereby proving the remaining inequality, in view of (2.1).

Now,

$$
\begin{aligned}
f_{1}\left(3 \nu^{2}\right)=3 \nu & \left(-9 \nu^{8}-99 \nu^{7}-402 \nu^{6}-76 \nu^{5}\right. \\
+ & \left.1128 \nu^{4}+608 \nu^{3}+2304 \nu^{2}+512 \nu-1536\right) .
\end{aligned}
$$

This polynomial, which has two positive zeros, is positive in between them, i.e., in $0.631229 \ldots<\nu<1.802069 \ldots$. In view of $(2.2), f_{1}\left(3 \nu^{2}\right)>0$ under the present hypotheses, which can be satisfied only if $0.7552<\nu<1$.

Further,

$$
\begin{aligned}
f_{1}\left(\nu^{2}+2\right)= & -\nu^{9}-3 \nu^{8}-56 \nu^{7}+258 \nu^{6}-1008 \nu^{5}-1188 \nu^{4} \\
& +7552 \nu^{3}+3576 \nu^{2}-2032 \nu+192 .
\end{aligned}
$$

This polynomial is positive in $0.227558 \ldots<\nu<2.606564 \ldots$, and hence for $0.7552<\nu<1$, the values which are relevant here.

This completes the proof.

Corollary. If $0<\nu<1$ and $\lambda<j_{\nu 1}$, then $J_{\nu}^{\prime}(\lambda)>0$.

Theorem 2.3. The following inequalities hold in the respective indicated intervals:

$$
\begin{array}{rlrl}
J_{\nu}^{\prime \prime \prime}(\nu) & <0, & 0.76 \leq \nu \leq 1 \\
J_{\nu}^{\prime \prime \prime}\left(\sqrt{\nu^{2}+1 / 3}\right) & <0, & 0.75563 & \leq \nu<1 \\
J_{\nu}^{\prime \prime \prime}\left(\sqrt{4.2 \nu^{2}-1.2}\right) & <0, & 0.78 \leq \nu<1 . \\
J_{\nu}^{\prime \prime \prime}(1) & <0, & 0.76 \leq \nu<1
\end{array}
$$

Proof. From (1.1) we find

$$
\begin{aligned}
& 2^{\nu}(6144) \Gamma(\nu+5) x^{3-\nu} J_{\nu}^{\prime \prime \prime}(x) \\
& <6144 \nu\left(\nu^{2}-1\right)\left(\nu^{2}-4\right)(\nu+3)(\nu+4)-1536 \nu(\nu+1)(\nu+2)^{2}(\nu+3)(\nu+4) x^{2} \\
& \quad+192(\nu+2)(\nu+3)^{2}(\nu+4)^{2} x^{4}-16(\nu+4)^{2}(\nu+5)(\nu+6) x^{6} \\
& \quad+(\nu+6)(\nu+7)(\nu+8) x^{8}
\end{aligned}
$$

When $x=\nu$, the resulting polynomial upper bound is negative for the stated interval. The same holds true for (2.5) and (2.7). But (2.6) is verified by this method only for $0.78 \leq \nu \leq 0.997 \ldots$. 
For the overlapping interval $0.82 \leq \nu<1$, (2.6) will be established via the differential equation (1.3). Putting $x^{2}=4.2 \nu^{2}-1.2$ in (1.3) gives the equation

$$
x^{3} J_{\nu}^{\prime \prime \prime}(x)+\left(\nu^{2}-1\right) 3.2 x J_{\nu}^{\prime}(x)+1.2\left(1-\nu^{2}\right) J_{\nu}(x)=0 .
$$

The sum of the last two terms will be shown to be positive for $0.82 \leq \nu<1$. This will complete the proof of (2.6).

This sum divided by the positive factor $\frac{5}{2}\left(1-\nu^{2}\right)$ equals

$$
3 J_{\nu}(x)-8 x J_{\nu}^{\prime}(x)=(3-8 \nu) J_{\nu}(x)+8 x J_{\nu+1}(x)
$$

from (1.4).

This last expression when divided by the positive factor $J_{\nu}(x)$ becomes

$$
\begin{aligned}
3-8 \nu+8 x \frac{J_{\nu+1}(x)}{J_{\nu}(x)} & =3-8 \nu+16 x^{2} \sum_{k=1}^{\infty} \frac{1}{j_{\nu k}^{2}-x^{2}} \\
& >3-8 \nu+16 x^{2} \sum_{k=1}^{\infty} \frac{1}{j_{\nu k}^{2}} \\
& =3-8 \nu+\frac{16\left(4.2 \nu^{2}-1.2\right)}{4(\nu+1)} \\
& =\frac{44 \nu^{2}-25 \nu-9}{5(\nu+1)}>0, \quad \nu \geq 0.82
\end{aligned}
$$

establishing (2.6).

The first infinite series introduced in this calculation is the standard partial fraction expansion [8, $\S 15.41(1), \mathrm{p} .498]$. The other is the familiar Rayleigh sum; it, together with its value, are found in $[8, \S 15.51$, p.502].

Remark. The partial sums of $J_{\nu}^{\prime \prime \prime}(x)$ are ineffective for (2.6) when $\nu$ is near 1 , because $J_{\nu}^{\prime \prime \prime}\left(\sqrt{4.2 \nu^{2}-1.2}\right)$ vanishes when $\nu=1$, as (1.3) shows.

Theorem 2.4. If $0.68177 \ldots<\nu<1$ and $\lambda \leq 3 \nu^{2}$, then

$$
3 \lambda^{4}-2\left(3 \nu^{2}+1\right) \lambda^{2}+3 \nu^{4}-10 \nu^{2}+4<0 .
$$

Moreover, this polynomial is an increasing function of $\lambda$ when $\lambda^{2}<\nu^{2}+\frac{1}{3}$ and a decreasing function when $\lambda^{2}>\nu^{2}+\frac{1}{3}$, $\nu$ fixed.

Proof. For the $\nu$-values under consideration, the constant term (i.e., the $\lambda$-free term) is negative. For these $\nu$, therefore, this quadratic in $\lambda^{2}$ is negative whenever $\lambda^{2}<3 \nu^{2}$ if it is negative for $\lambda^{2}=3 \nu^{2}$. When $\lambda^{2}=3 \nu^{2}$ the polynomial becomes $4\left(3 \nu^{2}-1\right)\left(\nu^{2}-1\right)$; this is negative in $0.58 \leq \nu<1$.

The final assertion of the theorem follows on differentiation with respect to $\lambda$. 


\section{Convexity and near-convexity of $J_{\nu}^{\prime \prime \prime}(x)$ and the number of $\lambda$ 's in $0<x<j_{\nu 1}$, for $x^{2} \leq 3 \nu^{2}, 0<\nu<1$}

There are, as will be demonstrated in this section, no more than two possible zeros of $J_{\nu}^{\prime \prime \prime}(x)$ in $0<x<j_{\nu 1}$ when $0<\nu<1$. It is already known [7, (4.2)] that there are none when $0<\nu \leq 0.7552$. Theorem 2.2 has established that, for any such zero, $\lambda^{2}<3 \nu^{2}$.

That there are no more than two $\lambda$ 's would be a consequence of $J_{\nu}^{\prime \prime \prime}(x)$ being convex in $x^{2}<3 \nu^{2}$. Indeed, this will be shown to be the case when $0<\nu \leq 0.877 \ldots$ Then, we shall learn that (i) $J_{\nu}^{\prime \prime \prime}(x)$ is convex in $0<x<1,0<\nu<1$, (ii) $J_{\nu}^{\prime \prime \prime}(x)$ vanishes (once) in $0<x<1,0.72127 \ldots<\nu<1$, say at $x=\lambda_{1}$, while (iii) $J_{\nu}^{(4)}(x)>0$, $1<x<\nu \sqrt{3}, 0<\nu<1$. All told, this shows that there can be no more than two zeros in $0<x<j_{\nu 1}, 0<\nu<1$.

Theorem 3.1. If $0<\nu<0.8771314 \ldots$, then $J_{\nu}^{\prime \prime \prime}(x)$ is convex in $0<x<\nu \sqrt{3}$.

Proof. From (1.1) we find, for $x^{2} \leq 3 \nu^{2}, 0<\nu<1$,

$$
\begin{aligned}
384 & \Gamma(\nu+2) x^{5-\nu} 2^{\nu} J_{\nu}^{(5)}(x) \\
> & 384 \nu\left(\nu^{2}-1\right)(\nu-2)(\nu-3)(\nu-4)-96 \nu\left(\nu^{2}-1\right)\left(\nu^{2}-4\right) x^{2} \\
& +12 \nu(\nu+1)(\nu+3)(\nu+4) x^{4}-(\nu+4)(\nu+5)(\nu+6) x^{6} \\
:= & f_{2}\left(x^{2}\right) .
\end{aligned}
$$

The cubic $f_{2}(x)$ achieves its minimum in $x \leq 3 \nu^{2}$ either at

$$
x_{0}=\frac{4 \nu(\nu+4)(\nu+3)-4 \sqrt{\nu(\nu+1)(\nu+4)\left(-\nu^{5}-9 \nu^{4}+13 \nu^{3}+209 \nu^{2}+188 \nu-240\right)}}{(\nu+4)(\nu+5)(\nu+6)}
$$

or at the end-point $x=3 \nu^{2}$.

The local minimum $f_{2}\left(x_{0}\right)$ is positive for all $0<\nu \leq 1$. However, the absolute minimum $f_{2}\left(3 \nu^{2}\right)$ is positive only for $0<\nu \leq 0.8771314 \ldots$, as asserted.

Corollary. The function $J_{\nu}^{\prime \prime \prime}(x)$ cannot have more than two zeros in $0<x<j_{\nu 1}$ when $0<\nu \leq 0.87713$. It has none there when $0<\nu \leq 0.7552$.

The last sentence restates $[7,(4.2)]$.

Theorem 3.2. If $0.013688 \leq \nu<1$, then $J_{\nu}^{\prime \prime \prime}(x)$ is convex in $0 \leq x \leq 1$.

Proof. Again, it will be shown that $J_{\nu}^{(5)}(x)>0$ for the relevant $\nu$ and $x$.

The absolute minimum of $f_{2}(x)$ occurs either at the local minimum $f_{2}\left(x_{0}\right)$ or at an end-point $(x=0, x=1)$.

First, we have $f_{2}(0)>0$, since $0<\nu<1$. Next, we recall from the proof of Theorem 3.1 that $f_{2}\left(x_{0}\right)>0,0<\nu<1$. Finally, putting $x=1$ causes $f_{2}(x)$ to become a polynomial in $\nu$ which vanishes at $\nu=0.013871 \ldots$ and at $\nu=1.09248 \ldots$ and is positive in between. This proves the theorem.

Corollary. If $0<\nu<1$, the function $J_{\nu}^{\prime \prime \prime}(x)$ cannot vanish more than twice in $0<x \leq 1$. 
Remark. Inequalities (2.1) and (2.7) imply further that $J_{\nu}^{\prime \prime \prime}(x)$ vanishes exactly once in $0<x \leq 1$ when $0.76<\nu<1$.

Theorem 3.3. If $0.72172 \ldots<\nu<1$ and $1 \leq x^{2}<3 \nu^{2}$, then $J_{\nu}^{(4)}(x)>0$ for $1<x<j_{\nu 1}$, and, in particular, $J_{\nu}^{(4)}(\lambda)>0$ for $1<\lambda<j_{\nu 1}$.

Proof. From (1.1), with $0<\nu<1, x^{2} \leq 3 \nu^{2}$,

$$
\begin{aligned}
& (384) 2^{\nu} \Gamma(\nu+3) x^{4-\nu} J_{\nu}^{(4)}(x) \\
& >384 \nu\left(\nu^{2}-1\right)\left(\nu^{2}-4\right)(\nu-3)-96 \nu\left(\nu^{2}-1\right)(\nu+2)^{2} x^{2} \\
& \quad+12(\nu+1)(\nu+2)(\nu+3)(\nu+4) x^{4}-(\nu+4)(\nu+5)(\nu+6) x^{6} \\
& \quad:=f_{3}\left(x^{2}\right) .
\end{aligned}
$$

The cubic $f_{3}(x)$ has a negative constant term, since $0<\nu<1$, and so has either two positive zeros or none. Hence, if $f_{3}(1)>0$ and $f_{3}\left(3 \nu^{2}\right)>0,0.72172 \ldots<\nu \leq 1$, then $f_{3}(x)>0,1 \leq x \leq 3 \nu^{2}, 0.72172 \ldots<\nu \leq 1$.

The polynomial $f_{3}(1)$ vanishes at $\nu=0.72172 \ldots$, next at $\nu=1.73768 \ldots$, and is positive in between. The polynomial $f_{3}\left(3 \nu^{2}\right)$ vanishes at $\nu=0.63122 \ldots$, next at $\nu=1.802096$, and is positive in between.

Thus, $J_{\nu}^{(4)}(x)>0,0.72172<\nu<1$, when $1 \leq x \leq \nu \sqrt{3}$. Inasmuch as $\lambda<\nu \sqrt{3}$ when $\lambda<j_{\nu 1}, 0<\nu<1$, the assertion is established.

Theorem 3.4. If $0<\nu<1$, then $J_{\nu}^{\prime \prime \prime}(x)$ cannot have more than two zeros in $0<x<j_{\nu 1}$.

Proof. The Corollary to Theorem 3.1 already establishes this when $0<\nu<$ $0.8771314 \ldots$ For the overlapping interval, $0.76<\nu<1$, the conclusion follows from Theorem 3.3 and the Corollary to Theorem 3.2.

Remark. There are exactly two such zeros when $0.76 \leq \nu<1$, according to (2.1) and (2.7). There are none when $0<\nu<0.7552$. Below it will be shown that $\nu=\nu_{0}=0.75558 \ldots$ is the value below which there are none and above which there are two.

\section{Monotonicity of the zeros}

The results of $\S 3$ show that monotonicity of the zeros of $J_{\nu}^{\prime \prime \prime}(x), 0<x<j_{\nu 1}, 0<\nu<1$, as a function of $\nu$, would follow on showing that $J_{\nu}^{\prime \prime \prime}(x)$ decreases (from zero) when $\nu$ is increased while keeping $x$ fixed at a value which causes $J_{\nu}^{\prime \prime \prime}(x)$ to vanish for the original $\nu$. Denoting that value by $\lambda_{\nu}$, or simply $\lambda$, this means demonstrating that

$$
\left.\frac{\partial J_{\nu}^{\prime \prime \prime}(x)}{\partial \nu}\right|_{x=\lambda_{\nu}}<0 .
$$

More precisely, this would show that, of the two zeros of $J_{\nu}^{\prime \prime \prime}(x)$ which could occur, the first would decrease and the second increase as $\nu$ increases to 1.

It would also show something else, namely, that there exists a unique value of $\nu$, say $\nu_{0}$, such that in $0<x<j_{\nu 1}, J_{\nu}^{\prime \prime \prime}(x)$ has no zeros when $0<\nu<\nu_{0}$, a double zero when $\nu=\nu_{0}$, and exactly two distinct simple zeros when $\nu_{0}<\nu<1$. We know already $[7,(4.2)]$ that $\nu_{0}>0.7552$. 
With the knowledge that $J_{\nu}^{\prime \prime \prime}(x)$ does not possess any zeros in $0<x<j_{\nu 1}$ when $0<\nu<0.7552$, the study of (4.1) when $\nu>0.755$ will lead to the desired results. It will show that whenever $\lambda$ exists for $\nu>0.755$, then (4.1) holds.

Here we need $[2, \S 7.2 .4(29)$, p.7]

$$
\frac{\partial J_{\nu}(x)}{\partial \nu}=J_{\nu}(x) \log \frac{1}{2} x-\frac{1}{2^{\nu}} \sum_{k=0}^{\infty} \frac{(-1)^{k} \psi(\nu+k+1) x^{2 k+\nu}}{4^{k} k ! \Gamma(\nu+k+1)}
$$

where $\psi(t)=\Gamma^{\prime}(t) / \Gamma(t)$. This function satisfies the recursion formula $[1, \S 6.3 .5, \mathrm{p} .258]$

$$
\psi(t+1)=\psi(t)+\frac{1}{t}
$$

and, it is important to bear in mind, is an increasing function of $t>1[1, \S 6.4 .1$, p.260].

The analyticity of $J_{\nu}(x)$ in both $\nu$ and $x$ permits us to infer from (4.2) that

$$
\begin{aligned}
& x^{3} \frac{\partial J_{\nu}^{\prime \prime \prime}(x)}{\partial \nu}=x^{3} J_{\nu}^{\prime \prime \prime}(x) \log \frac{1}{2} x+3 x^{2} J_{\nu}^{\prime \prime}(x)-3 x J_{\nu}^{\prime}(x)+2 J_{\nu}(x) \\
&-\frac{x^{\nu}}{2^{\nu}} \sum_{k=0}^{\infty} \frac{(-1)^{k}(\nu+2 k-2)(\nu+2 k-1)(\nu+2 k) \psi(\nu+k+1) x^{2 k}}{4^{k} k ! \Gamma(\nu+k+1)}
\end{aligned}
$$

When $x$ is chosen to be a zero, $\lambda$, of $J_{\nu}^{\prime \prime \prime}(x)$, this simplifies considerably on application of Bessel's differential equation (1.2) and of the related differential equation (1.3) which permits expressing $J_{\nu}^{\prime}(\lambda)$ in terms of $J_{\nu}(\lambda)$.

This done, we have

$$
\begin{aligned}
& \left.x^{3} \frac{\partial J_{\nu}^{\prime \prime \prime}(x)}{\partial \nu}\right|_{x=\lambda}=\frac{3 \lambda^{4}-2\left(3 \nu^{2}+1\right) \lambda^{2}+3 \nu^{4}-10 \nu^{2}+4}{\nu^{2}+2-\lambda^{2}} J_{\nu}(\lambda) \\
& -\frac{\lambda^{2}}{2^{\nu}} \sum_{k=0}^{\infty} \frac{(-1)^{k}(\nu+2 k-2)(\nu+2 k-1)(\nu+2 k) \psi(\nu+k+1) \lambda^{2 k}}{4^{k} k ! \Gamma(\nu+k+1)} .
\end{aligned}
$$

The coefficient of $J_{\nu}(\lambda)$ in (4.5) is negative when $0.68177 \ldots<\nu<1$, according to Theorem 2.4 and (2.3). The algebraic value of the first term in (4.5) is increased, therefore, if the positive factor $J_{\nu}(\lambda)$ is decreased. From (1.1)

$$
J_{\nu}(\lambda)>\frac{\lambda^{\nu}}{2^{\nu}} \frac{1}{\Gamma(\nu+1)}\left[1-\frac{\lambda^{2}}{4(\nu+1)}\right] .
$$

Hence, for $0.681777 \ldots<\nu<1$,

$$
\left.2^{\nu} \Gamma(\nu+1) \lambda^{3-\nu} \frac{\partial J_{\nu}^{\prime \prime \prime}(x)}{\partial \nu}\right|_{x=\lambda}<\alpha-\beta
$$

where

$$
\alpha=\frac{\left(4 \nu+4-\lambda^{2}\right)\left[3 \lambda^{4}-2\left(3 \nu^{2}+1\right) \lambda^{2}+3 \nu^{4}-10 \nu^{2}+4\right]}{4(\nu+1)\left(\nu^{2}+2-\lambda^{2}\right)}
$$


and, using (4.4) and (4.3),

$$
\begin{aligned}
\beta= & \nu(\nu-1)(\nu-2) \psi(\nu+1)-\frac{1}{4} \nu(\nu+2)\left[\psi(\nu+1)+\frac{1}{\nu+1}\right] \lambda^{2} \\
& +\frac{(\nu+3)(\nu+4)\left[\psi(\nu+1)+\frac{1}{\nu+1}+\frac{1}{\nu+2}\right] \lambda^{4}}{32(\nu+1)} \\
& -\frac{(\nu+4)(\nu+5)(\nu+6)\left[\psi(\nu+1)+\frac{1}{\nu+2}+\frac{1}{\nu+3}\right] \lambda^{6}}{384(\nu+1)(\nu+2)(\nu+3)} .
\end{aligned}
$$

The coefficient of the quartic polynomial in $\alpha$ is a positive increasing function of $\lambda, \lambda^{2}<3 \nu^{2}$. The polynomial itself, which is negative (Theorem 2.4), decreases in $\lambda^{2}<\nu^{2}+\frac{1}{3}$ and increases in $\nu^{2}+\frac{1}{3}<\lambda^{2}<3 \nu^{2}$. We consider these two cases separately:

(A) $\lambda^{2} \leq \nu^{2}+\frac{1}{3}$. (This certainly occurs when $0.75563 \leq \nu<1$, from (2.5)). Under these circumstances $\lambda=\lambda_{1}<\lambda_{2}$.

Keeping in mind that $\psi(t)$ increases when $t>1$,

$$
\begin{gathered}
\alpha-\beta<\frac{(4 \nu+4-m)\left[3 m^{2}-2\left(3 \nu^{2}+1\right) m+3 \nu^{4}-10 \nu^{2}+4\right]}{4(\nu+1)\left(\nu^{2}+2-m\right)} \\
-\nu(\nu-1)(\nu-2) P+\frac{1}{4} \nu(\nu+2)\left[Q+\frac{1}{\nu+1}\right] M \\
-\frac{(\nu+3)(\nu+4)\left[P+\frac{1}{\nu+1}+\frac{1}{\nu+2}\right] m^{2}}{32(\nu+1)} \\
+\frac{(\nu+4)(\nu+5)(\nu+6)\left[Q+\frac{1}{\nu+1}+\frac{1}{\nu+2}+\frac{1}{\nu+3}\right] M^{3}}{384(\nu+1)(\nu+2)(\nu+3)} .
\end{gathered}
$$

The quantities $m, M, P$, and $Q$ may be assigned any values for which

$$
\begin{array}{ll}
0<m \leq \min \lambda^{2}, & M \geq \max \lambda^{2}, \\
0<P \leq \min \psi(\nu+1), & Q \geq \max \psi(\nu+1),
\end{array}
$$

where $\nu$ varies over the particular interval under consideration.

The first of two $\nu$-intervals to be considered here is (i) $0.75 \leq \nu \leq 0.995$. Here, let $m=1-\nu$, as is permissible according to [7, (4.2)], and $M=\nu^{2}+\frac{1}{3}$. Further, from $[1, \mathrm{p} .270]$,

$$
P=0.24<\psi(1.75) \text { and } Q=0.42>\psi(1.995) .
$$

With these values, (4.9) can be rewritten to provide a polynomial upper bound in the form, for $0.75 \leq \nu \leq 0.995$,

$$
\begin{aligned}
& 10368(\nu+1)^{2}(\nu+2)^{2}(\nu+3)^{2}\left(\nu^{2}+2-m\right)(\alpha-\beta) \\
& <11.34 \nu^{14}+330.48 \nu^{13}+4953.96 \nu^{12}+40014.54 \nu^{11}+237710.16 \nu^{10} \\
& \quad+1214298 \nu^{9}+4568979.84 \nu^{8}+10335097.8 \nu^{7}+10653286.14 \nu^{6} \\
& \quad-3123780.64 \nu^{5}-17944967.56 \nu^{4}-12929826.42 \nu^{3}+1321292.68 \nu^{2} \\
& \quad+4486314.88 \nu+1157758.08 .
\end{aligned}
$$

This polynomial has two positive zeros, $0.58979 \ldots$ and $0.99968 \ldots$, in between which it is negative. For $\lambda^{2} \leq \nu^{2}+\frac{1}{3}$, this establishes (4.1) when $0.75 \leq \nu \leq 0.995$. 
To complete (A), we consider the remaining interval (ii) $0.995 \leq \nu<1$. Here the zeros $\lambda_{1}, \lambda_{2}$ are treated separately. We have $\lambda_{1}^{2}<\nu^{2}<\lambda_{2}^{2} \leq \nu^{2}+\frac{1}{3}$, since $J_{\nu}^{\prime \prime \prime}(\nu)<0$ from (2.4) and (2.1). For $\lambda_{1}$, we may therefore let $m=1-\nu$ as before, but now with $M=\nu^{2}$. Let $P=0.41<\psi(1.995)$ and $Q=0.43>\psi(2)$.

The polynomial bound corresponding to the one in (i) has two positive zeros, $0.53684 \ldots, 1.06377 \ldots$, and is negative in between, establishing (4.1) for $\lambda=\lambda_{1}$ and $0.995 \leq \nu \leq 1$.

For $\lambda_{2}$, we may take $m=\nu^{2}, M=\nu^{2}+\frac{1}{3}$, again with $P=0.41$ and $Q=0.43$. With these values as well, the polynomial upper bound corresponding to the one in (i) is negative for $0.995 \leq \nu \leq 1$ (in fact, even for $0.63014 \ldots<\nu<1.01405 \ldots$ ). Thus, (4.1) is verified for $0.75 \leq \nu<1$, when $\lambda^{2} \leq \nu^{2}+\frac{1}{3}$.

(B) $\nu^{2}+\frac{1}{3} \leq \lambda^{2}<3 \nu^{2}$. Here $\alpha$ is bounded above by assigning $\lambda^{2}$ a lower bound $m$ in the factor $\left(4 \nu+4-\lambda^{2}\right) /\left(\nu^{2}+2-\lambda^{2}\right)$ and an upper bound $M$ in the polynomial

$$
3 \lambda^{4}-2\left(3 \nu^{2}+1\right) \lambda^{2}+3 \nu^{4}-10 \nu^{2}+4,
$$

since the polynomial increases in $\lambda^{2}>\nu^{2}+\frac{1}{3}$ and is negative for the values of $\lambda$ and $\nu$ under study. This done,

$$
\begin{gathered}
\alpha-\beta<\frac{1}{4(\nu+1)} \frac{4 \nu+4-m}{\nu^{2}+2-m}\left[3 M^{2}-2\left(3 \nu^{2}+1\right) M^{2}+3 \nu^{4}-10 \nu^{2}+4\right] \\
-\nu(\nu-1)(\nu-2) P+\frac{\nu(\nu+2)}{4}\left[Q+\frac{1}{\nu+1}\right] M \\
-\frac{(\nu+3)(\nu+4)\left[P+\frac{1}{\nu+1}+\frac{1}{\nu+2}\right] m^{2}}{32(\nu+1)} \\
+\frac{(\nu+4)(\nu+5)(\nu+6)\left[Q+\frac{1}{\nu+1}+\frac{1}{\nu+2}+\frac{1}{\nu+3}\right] M^{3}}{384(\nu+1)(\nu+2)(\nu+3)} .
\end{gathered}
$$

The quantities $m, M, P, Q$ retain the significances assigned to them in part (A). Once these quantities are selected appropriately, we have again a polynomial upper bound for

$$
10368(\nu+1)^{2}(\nu+2)^{2}(\nu+3)^{2}\left(\nu^{2}+2-m\right)(\alpha-\beta) .
$$

The proof that $\alpha-\beta<0$, and hence that $\partial J_{\nu}^{\prime \prime \prime}(x) / \partial \nu<0$ at $x=\lambda$ when $\nu^{2}+\frac{1}{3} \leq \lambda^{2}<3 \nu^{2}$, is divided into two parts.

(i) $0.755 \leq \nu \leq 0.78$. Here we use $m=\nu^{2}+\frac{1}{3}, M=3 \nu^{2}, P=0.2512<\psi(1.755)$, $Q=0.2702>\psi(1.78)$. The resulting polynomial upper bound for (4.10) vanishes at $\nu=0.61744 \ldots$, next at $\nu=0.78045 \ldots$, and is negative in between. This verifies the assertion for $0.755 \leq \nu \leq 0.78$.

(ii) $0.78 \leq \nu<1$. Here closer bounds on $\lambda$ are needed. In (2.5) it is recorded that $J_{\nu}^{\prime \prime \prime}\left(\sqrt{\nu^{2}+1 / 3}\right)<0,0.7556 \ldots \leq \nu<1$. Thus, there are two zeros, $\lambda_{1}, \lambda_{2}$, of $J_{\nu}^{\prime \prime \prime}(x)$ in $0<x<j_{\nu 1}$ when $0.78 \leq \nu<1$, in view of (2.1). Clearly, $\lambda_{1}^{2}<\nu^{2}+\frac{1}{3}$, and so falls into part (A) of this proof.

It remains only to consider $\lambda_{2}$. From (2.6) and (2.1), it is immediate that $\lambda_{2}^{2}>4.2 \nu^{2}-1.2,0.78 \leq \nu<1$. Now we choose $m=4.2 \nu^{2}-1.2, M=3 \nu^{2}$, $P=0.27<\psi(1.78), Q=0.43>\psi(2)$. 
This done, the resulting polynomial upper bound for (4.10) vanishes at $\nu=$ $0.69327 \ldots$, next at $\nu=2.75071 \ldots$, and is negative in between.

In sum, if $\lambda$ is a zero of $J_{\nu}^{\prime \prime \prime}(x)$ and if $0.755 \leq \nu<1$, then

$$
\left.\frac{\partial J_{\nu}^{\prime \prime \prime}(x)}{\partial \nu}\right|_{\lambda}<0 .
$$

This inequality, combined with the results of $\S 3$, yield the following (keeping in mind that there are no zeros of $J_{\nu}^{\prime \prime \prime}(x)$ in $0<x<j_{\nu 1}$ when $\left.\nu \leq 0.7552\right)$.

Theorem 4.1. If $\lambda_{1}, \lambda_{2}$ are zeros of $J_{\nu}^{\prime \prime \prime}(x), 0<\nu<1, \lambda_{1}<\lambda_{2}<j_{\nu 1}$, then $\lambda_{1}$ is a steadily decreasing function of $\nu$ as $\nu$ increases to 1 and $\lambda_{2}$ is a steadily increasing function of $\nu$ as $\nu$ increases to 1 . Further, there exists a (unique) value of $\nu=\nu_{0}$ such that $J_{\nu}^{\prime \prime \prime}(x)$ has two zeros when $\nu_{0}<\nu<1$ and none when $0<\nu<\nu_{0}$. When $\nu=\nu_{0}$, $J_{\nu}^{\prime \prime \prime}(x)$ has a double zero in $0<x<j_{\nu 1}$.

Remarks. 1. Kerimov and Skorokhodov $[3,4]$ have studied the multiple zeros of Bessel functions and their first three derivatives. In particular, they have found an algebraic equation connecting the order $\nu$ and the argument of a double zero [3, (2.5)].

2. Martin Muldoon has determined, using Maple V.1, that $\nu_{0}=0.7555783929$ and $\lambda_{1}=\lambda_{2}=0.9593887612$. These values were found as the common zero of $J_{\nu}^{\prime \prime \prime}(x)$ and $J_{\nu}^{(4)}(x)$ for $0<\nu<1$. This is the device used also in [3,4], using different computer facilities.

Theorem 4.2. Let $\lambda_{1}(\nu)<\lambda_{2}(\nu)$ denote the zeros of $J_{\nu}^{\prime \prime \prime}(x)$ in $0<x<j_{\nu 1}$, $\nu_{0}<\nu<1$. Then $\lambda_{1}(\nu) \downarrow 0, \lambda_{2}(\nu) \uparrow \sqrt{3}=j_{11}^{\prime \prime \prime}$ as $\nu \uparrow 1$.

Proof. The respective limits, $\Lambda_{1}, \Lambda_{2}$, clearly exist, since $\lambda_{1}(\nu), \lambda_{2}(\nu)$ are bounded monotonic functions.

From (1.3),

$$
\lambda_{i}(\nu) J_{\nu}^{\prime}\left(\lambda_{i}\right)=\frac{3 \nu^{2}-\lambda_{i}^{2}(\nu)}{\nu^{2}+2-\lambda_{i}^{2}(\nu)} J_{\nu}\left(\lambda_{i}(\nu)\right), \quad i=1,2 \ldots
$$

Therefore, letting $\nu \rightarrow 1$, since $\Lambda_{1} \leq \lambda_{1}(0.76)=0.87109<\sqrt{3}$,

$$
\Lambda_{1} J_{1}^{\prime}\left(\Lambda_{1}\right)=\frac{3-\Lambda_{1}^{2}}{3-\Lambda_{1}^{2}} J_{1}\left(\Lambda_{1}\right)=J_{1}\left(\Lambda_{1}\right),
$$

and so, from (1.4),

$$
J_{1}\left(\Lambda_{1}\right)=\Lambda_{1} J_{1}^{\prime}\left(\Lambda_{1}\right)=-\Lambda_{1} J_{2}\left(\Lambda_{1}\right)+J_{1}\left(\Lambda_{1}\right) .
$$

Therefore, $\Lambda_{1} J_{2}\left(\Lambda_{1}\right)=0$, so that $\Lambda_{1}=0$, since $\Lambda_{1}<\sqrt{3}<j_{21}$.

If $\Lambda_{2}^{2} \neq 3$, then (4.12) would imply, as above, that $\Lambda_{2}=0$. However, $\Lambda_{2}>$ $\lambda_{2}(0.76)>1$. Hence $\Lambda_{2}=\sqrt{3}$, as asserted.

Remark. Theorems 4.1 and 4.2 suggest that, for each fixed $n=1,2,3, \ldots$, there exists a unique value $\nu_{n}, n-3<\nu_{n}<n-2$, corresponding to which there are precisely two zeros, $\lambda_{\nu 1}^{(n)}, \lambda_{\nu 2}^{(n)}$, of $J_{\nu}^{(n)}(x)$ in $0<x<j_{\nu 1}$, where $\nu_{n}<\nu<n-2$, and that, as $\nu \uparrow n-2$, we have $\lambda_{\nu 1}^{(n)} \downarrow 0, \lambda_{\nu 2}^{(n)} \uparrow j_{n-2,1}^{(n)}$. For $n-3<\nu<\nu_{n}$, these zeros would not exist, according to the conjecture. Theorems 4.1 and 4.2 verify the case $n=3$. For $n=2$, the conjecture is verified in [6]. It holds also for $n=1[5]$. For $n=4,5, \ldots, 13$, calculations using Maple V.1 have produced strong numerical support. 


\section{Inequalities}

Bounds for the zeros of $J_{\nu}^{\prime \prime \prime}(x)$ in $0<x<j_{\nu 1}$ can be generated by a method applicable also to such zeros of $J_{\nu}^{(n)}(x), n=2,4,5, \ldots$. They arise from a combination of the differential equation (1.3) and the expansion $[2, \S 7.9(4)$, p. 61$]$

$$
\frac{x J_{\nu+1}(x)}{J_{\nu}(x)}=2 \sum_{k=1}^{\infty} \sigma_{\nu}^{(k)} x^{2 k}, \quad|x|<j_{\nu 1}
$$

where the coefficients are the Rayleigh sums [8, §15.51, p. 502]

$$
\sigma_{\nu}^{(k)}:=\sum_{m=1}^{\infty} \frac{1}{j_{\nu m}^{2 k}}
$$

In particular $[8, \S 15.51$, p. 502],

$$
\begin{gathered}
\sigma_{\nu}^{(1)}=\frac{1}{4(\nu+1)}, \quad \sigma_{\nu}^{(2)}=\frac{1}{16(\nu+1)^{2}(\nu+2)}, \\
\sigma_{\nu}^{(3)}=\frac{1}{32(\nu+1)^{3}(\nu+2)(\nu+3)} .
\end{gathered}
$$

The recursion formula (1.4) permits rewriting (5.1), for $|x|<j_{\nu 1}$, as

$$
\frac{x J_{\nu}^{\prime}(x)}{-J_{\nu}(x)}=\nu-2 \sum_{k=1}^{\infty} \sigma_{\nu}^{(k)} x^{2 k}, \quad|x|<j_{\nu 1}
$$

With $x=\lambda$, this becomes, in view of (1.3),

$$
(\nu-1) \frac{\nu^{2}-2 \nu-\lambda^{2}}{\nu^{2}+2-\lambda^{2}}=2 \sum_{k=1}^{\infty} \sigma_{\nu}^{(k)} \lambda^{2 k}, \quad 0<\lambda<j_{\nu 1} .
$$

Hence, for each $n=1,2, \ldots$,

$$
(\nu-1) \frac{\nu^{2}-2 \nu-\lambda^{2}}{\nu^{2}+2-\lambda^{2}}>2 \sum_{k=1}^{n} \sigma_{\nu}^{(k)} \lambda^{2 k}, \quad 0<\lambda<j_{\nu 1} .
$$

The first three inequalities are, in view of (5.2), for $0<\nu<1$ and $\lambda<j_{\nu 1}$,

$$
\begin{gathered}
\lambda^{4}-3 \nu^{2} \lambda^{2}+2 \nu\left(\nu^{2}-1\right)(\nu-2)>0, \\
\lambda^{6}+3\left(\nu^{2}+4 \nu+2\right) \lambda^{4}-12 \nu^{2}(\nu+1)(\nu+2) \lambda^{2} \\
+8 \nu\left(\nu^{2}-1\right)\left(\nu^{2}-4\right)(\nu+1)>0,
\end{gathered}
$$

and

$$
\begin{aligned}
\lambda^{8}+ & \left(\nu^{2}+8 \nu+4\right) \lambda^{6}+6(\nu+1)(\nu+3)\left(\nu^{2}+4 \nu+2\right) \lambda^{4} \\
& -24 \nu^{2}(\nu+1)^{2}(\nu+2)(\nu+3) \lambda^{2} \\
& +16 \nu(\nu-1)(\nu-2)(\nu+1)^{3}(\nu+2)(\nu+3)>0 .
\end{aligned}
$$

Of course, these inequalities apply only for those values of $\nu$ for which $\lambda$ exists, i.e., for $\nu_{0}<\nu<1, \nu_{0}=0.7555783929$. 
When $\nu>1, \lambda<j_{\nu 1}$, these inequalities are reversed. When $\nu=1$, each polynomial vanishes, since then the only $0<\lambda<j_{11}$ is $\lambda=\sqrt{3}$. With $\nu=1$ and $\lambda=0$, each polynomial also vanishes.

In principle, each of (5.5), (5.6), (5.7) can be solved for $\lambda$, since they are, respectively, quadratic, cubic, and quartic in $\lambda^{2}$. Thus, (5.5) (again with $\lambda_{1}<\lambda_{2}<$ $j_{\nu 1}$ ) leads to

$$
\begin{gathered}
\lambda_{1}^{2}<\frac{3 \nu^{2}-\sqrt{\nu^{4}+16 \nu^{3}+8 \nu^{2}-16 \nu}}{2}, \\
3 \nu^{2}>\lambda_{2}^{2}>\frac{3 \nu^{2}+\sqrt{\nu^{4}+16 \nu^{3}+8 \nu^{2}-16 \nu}}{2}
\end{gathered}
$$

when $0.7670046 \ldots<\nu<1$. For smaller values of $\nu$, the polynomial in (5.5) is positive for all $\lambda$ and so the inequalities (5.4) and (5.8) are vacuous.

For zeros of $J_{\nu}^{(n)}(x)$, for other $n$, this same approach yields analogous bounds. To locate them, it suffices to utilize the differential equation satisfied by $y=J_{\nu}(x)$

$$
x^{n} y^{(n)}+x p_{n}(x) y^{\prime}+q_{n}(x) y=0
$$

where $p_{n}(x)$ and $q_{n}(x)$ are polynomials (not necessarily of degree $n$ ), in place of (1.3). This will replace the right side of (5.3) and then lead to inequalities paralleling (5.4), (5.6), and (5.7).

In particular, when $n=2$, the Bessel equation (1.2), used instead of (1.3), would generate corresponding inequalities.

Thus, for the first positive zero, $j_{\nu 1}^{\prime \prime}$, of $J_{\nu}^{\prime \prime}(x), \nu>1$, already the first term of the series in (5.3) gives the inequality

$$
j_{\nu 1}^{\prime \prime 2}>\frac{2(\nu+1)}{2 \nu+1}\left(\nu^{2}-\nu\right), \quad \nu>1
$$

a sharpening of the lower bound in $[8, \S 5.3(6)$, p. 487].

The zeros of the polynomials in (5.5), (5.6), and (5.7) provide quite good approximations to $\lambda_{1}$ and $\lambda_{2}$ in $\nu_{0}<\nu<1$ from above and below, respectively. The accuracy increases as $\nu$ increases to 1 . This is illustrated by Table 1 which records these pairs of zeros of the polynomials in (5.5) and (5.7), along with the values of $\lambda_{1}, \lambda_{2}$ which Martin Muldoon kindly calculated using Maple V.1.

The polynomial in (5.5) is quadratic in $\lambda^{2}$. In (5.7) it is quartic in $\lambda^{2}$. The pertinent columns of pairs of zeros are labelled "quadratic" and "quartic" to emphasize this. A column labelled "cubic", recording the zeros of the polynomials, cubic in $\lambda^{2}$, in (5.6), would provide results intermediate between those found in the quadratic and quartic columns.

Polynomials of higher degree can be obtained from (5.4), since $\sigma_{\nu}^{(k)}$ has been determined explicitly for several further values of $k$, e.g., in $[8, \S 15.51$, p. 502]. These would provide even more accurate approximations to $\lambda_{1}$ and $\lambda_{2}$.

All of these polynomial approximations can be calculated on a variety of hand calculators.

Acknowledgments Alfred Gray, as mentioned in [7], constructed graphics using Mathematica which depict the monotonicity properties established both in [7] and here. His diagrammes led to the conjectures established in $\S 4$ here. As noted in the text, Martin E. Muldoon has kindly calculated many quantities recorded there. I am grateful also for other calculational help: David R. Stoutemyer (Soft 


\begin{tabular}{|l|cc|cc|cc|}
\hline \multicolumn{1}{|c|}{$\nu$} & \multicolumn{2}{|c|}{ quadratic } & \multicolumn{2}{c|}{ quartic } & \multicolumn{2}{c|}{ Maple V.1 } \\
\hline 0.76 & \multicolumn{2}{|c|}{ (no zeros) } & 0.871230863 & 1.04826120 & 0.8708922024 & 1.049281526 \\
0.78 & \multicolumn{2}{|c|}{ (no zeros) } & 0.750787863 & 1.174426317 & 0.750735024 & 1.175174705 \\
0.8 & 0.692820 & 1.689217 & 0.675505517 & 1.254413745 & 0.6754869619 & 1.255129498 \\
0.82 & 0.624087 & 1.275819 & 0.613575389 & 1.319896285 & 0.6135676964 & 1.320590339 \\
0.84 & 0.564937 & 1.340763 & 0.558131591 & 1.377558456 & 0.5581282251 & 1.378224647 \\
0.86 & 0.510654 & 1.394296 & 0.506149076 & 1.430177139 & 0.5061475983 & 1.430805016 \\
0.88 & 0.458790 & 1.453516 & 0.455812960 & 1.479216669 & 0.4558123326 & 1.479794224 \\
0.9 & 0.407650 & 1.504599 & 0.405724878 & 1.525558088 & 0.4057246298 & 1.526072611 \\
0.92 & 0.355685 & 1.553282 & 0.354495734 & 1.569778624 & 0.3544956468 & 1.570217036 \\
0.94 & 0.301003 & 1.600061 & 0.300328969 & 1.612279839 & 0.3003289444 & 1.612628852 \\
0.96 & 0.240482 & 1.645286 & 0.240159048 & 1.653353864 & 0.2401590438 & 1.653600060 \\
0.98 & 0.166567 & 1.689217 & 0.1664658955 & 1.693220727 & 0.1664658953 & 1.693350603 \\
1 & 0 & $\sqrt{3}$ & 0 & $\sqrt{3}$ & 0 & $\sqrt{3}$ \\
\hline
\end{tabular}

TABLE 1.

Warehouse, Honolulu) checked, using "Derive", the values I obtained for the zeros of the polynomial in (5.6). S. Adams, K. O. Geddes, and Daniel Dubois, all working with Maple V.2, made a variety of calculations overlapping those of Martin E. Muldoon. Some bugs were discovered in Maple V.2 as a result, since corrected in a later release.

Finally, Jason I. Brown provided very valuable help by performing symbolic algebraic manipulation on Maple V.1. These yielded the polynomials cited in the proof contained in $\S 4$.

\section{References}

1. M. Abramowitz and I. A. Stegun (Editors), Handbook of Mathematical Functions with Formulas, Graphs and Mathematical Tables, National Bureau of Standards, Applied Mathematics Series, 55, Washington, D. C., 1972.

2. A. Erdélyi (Ed.), Higher Transcendental Functions (Bateman Manuscript Project) v.2, McGrawHill Book Co. New York, Toronto, London, 1953.

3. M. K. Kerimov and S. L. Skorokhodov, Calculation of the multiple zeros of the derivatives of the cylindrical Bessel functions $J_{\nu}(z)$ and $Y_{\nu}(z)$, USSR Comput. Maths. Math. Phys. 25, 6 (1985), 101-107.

4. __ On multiple zeros of derivatives of Bessel's cylindrical functions, Soviet Math. Dokl. 33 (1986), 650-653.

5. L. Lorch and M. Muldoon, The zeros of the derivatives of Bessel functions of orders between -2 and -1 (preprint).

6. L. Lorch, M. Muldoon, and P. Szego, The points of inflection of Bessel functions of negative order, Canad. J. Math. 43 (1991), 1309-1322.

7. L. Lorch and P. Szego, Monotonicity of the zeros of the third derivative of Bessel functions. Methods Appl. Anal. 2 (1995), pp. 103-111.

8. G. N. Watson, A Treatise on the Theory of Bessel functions, 2nd ed. The University Press. Cambridge, 1944.

Department of Mathematics and Statistics, York University, North York, Ontario, CANADA M3J 1P3

E-mail: lorch@mathstat.yorku.ca 